\title{
Rapid Detection of Specific Gram-negative Microorganisms Causing Bloodstream Infections in Children with the Microarray Method
}

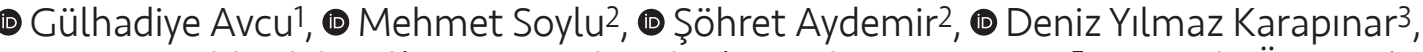

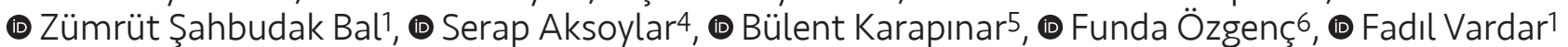 \\ ${ }^{1}$ Ege University Faculty of Medicine, Department of Pediatrics, Division of Pediatric Infectious Diseases, İzmir, Turkey \\ 2Ege University Faculty of Medicine, Department of Medical Microbiology, İzmir, Turkey \\ ${ }^{3}$ Ege University Faculty of Medicine, Department of Pediatrics, Division of Pediatric Hematology, İzmir, Turkey \\ ${ }^{4}$ Ege University Faculty of Medicine, Department of Pediatrics, Division of Pediatric Oncology and Bone Marrow Transplantation, Izmir, Turkey \\ ${ }^{5}$ Ege University Faculty of Medicine, Department of Pediatrics, Division of Pediatric Intensive Care, İzmir, Turkey \\ ${ }^{6}$ Ege University Faculty of Medicine, Department of Pediatrics, Division of Pediatric Gastroenterology, Hepatology and Nutrition, İzmir, Turkey
}

\begin{abstract}
Aim: Bloodstream infections are serious conditions that can cause significant morbidity and mortality. Early recognition of the bacteria and targeted early treatment play an important role in prognosis. The Verigene Gram-negative blood culture nucleic acid test (BC-GN; Nanosphere, USA) is a new method that can accurately identify both Gram-negative bacteria and resistance genes within 2 hours. In this study, we aimed to determine the accuracy of this assay for the rapid identification of certain Gram-negative microorganisms in children with bloodstream infections and to compare with the conventional culture method.

Materials and Methods: A total of 30 patients (<18 years-old) (followed up in the Department of Pediatrics of Ege University Faculty of Medicine between December 2015-June 2016) with bloodstream infections due to Gram-negative bacteria were prospectively included. Microarray test results were compared with conventional blood culture results.

Results: Klebsiella spp. (40\%) were the most common and Acinetobacter spp. (20\%) were the second most commonly seen Gram-negative microorganisms detected. Accurate identification via microarray analysis was 100\% (12/12) for Klebsiella spp, 100\% (6/6) for Acinetobacter spp., $100 \%$ (5/5) for Escherichia coli, 80\% (4/5) for Enterobacter cloacae spp., 100\% (1/1) for Pseudomonas aeruginosa and 100\% (1/1) for Citrobacter amalonaticus, respectively. The test was able to correctly identify $96.5 \%$ of Gram-negative microorganisms that were located in the test panel. CTX-M was the most common gene responsible for resistance.

Conclusion: The Verigene Gram-negative (BC-GN) microarray test (Nanosphere) is a rapid method which can correctly identify the located Gram-negative microorganisms in the panel and give results much faster than conventional methods.

Keywords: Bloodstream infection, child, gram-negative, microarray assay
\end{abstract}

\section{Introduction}

Bloodstream infections (BSIs) are serious conditions that can cause significant mortality and morbidity.
Hospital-acquired BSI caused by Gram-negative bacteria are increasing day by day and serious infections caused by multidrug-resistant bacteria are emerging (1). Moreover, 
delayed initiation of effective antimicrobial therapy has been associated with adverse outcomes in ExtendedSpectrum Beta-Lactamase (ESBL) positive bacteremia $(2,3)$. The risk of death from sepsis increases by $6 \%$ to $10 \%$ per hour (4). For this reason, besides the gold standard of classical culture methods, many rapid diagnostic tests have begun to be used. Blood culture is accepted as the most valuable method in the diagnosis of BSIs (5). However, the need for a 24-48-hour period for isolation, identification and susceptibility testing of the bacteria in blood culture is a significant disadvantage. Traditional culture methods also have limitations such as false positive results in cases of contamination that can result in unneeded antimicrobial treatment.

The Verigene Gram-negative blood culture nucleic acid test (BC-GN; Nanosphere, USA) is a new, automated, multiplexed nucleic acid test which is designed to detect most common Gram-negative bacteria and the resistance genes from classical blood cultures within approximately 2 hours of signal positivity. With this method, Acinetobacter spp., Klebsiella pneumoniae, Klebsiella oxytoca, Pseudomonas aeruginosa, Escherichia coli, Serratia marcescens, Proteus spp., Citrobacter spp., Enterobacter spp. and 6 resistance genes (CTX-M, KPC, NDM, VIM, IMP, OXA) can be identified. It is thought that the microarray method may play a role in empirical treatment of bacteremia in the development of more accurate and effective antibiotic therapy than conventional methods (6).

In this study; we aimed to determine the accuracy of this assay for the rapid identification of certain Gram-negative microorganisms in children with BSIs and to compare with the conventional culture method.

\section{Materials and Methods}

We prospectively followed and evaluated the data of patients aged 0-18 years, who were admitted to the Departments of Pediatrics, pediatric emergency care unit, neonatal and pediatric intensive care units (ICUs) of Ege University School of Medicine with the presumed diagnosis of BSI. Among them, a total of 30 patients whose blood cultures had a Gram-negative bacteria isolation with classic culture methods were again included for further study by the microarray method. The demographic properties of the patients (age, gender, comorbid diseases and ward admitted to) and the presence of predisposing factors for Gramnegative bacteremia (central venous line, total parenteral nutrition, surgical intervention, burn, immunosuppression, etc.) were recorded.
With the decision of the Board of Ethics of Ege University, dated 15.04.2015 and numbered 15-3.2 / 7, permission was obtained for this study. Informed consent was obtained from the patients included in the study.

\section{Microbiology}

In this study, the blood culture sample, after a positive signal with the conventional method, was first inoculated on to $5 \%$ sheep blood and Eosin Methylene-blue (EMB) agars. Gram staining results were then noted. From the Gram-negative colonies after 24-hour incubation, bacteria were identified with the Matrix-Assisted Laser Desorption/ Ionization Time-of-Flight Mass Spectrometry (MALDI-TOF MS) method. Following this, antibiotic sensitivities of the bacteria were identified with the $\operatorname{VITEK}^{\circledR} 2^{\text {TM }}$ (bioMérieux) method.

Finally, after closed system nucleic acid extraction and amplification of a $0.35 \mathrm{~mL}$ sample taken from the same blood culture bottles, Gram-negative bacteria and some of the resistant genes were identified with nanoparticle probes in a microarray test method. The results obtained from the conventional culture method were compared with those obtained from the microarray method.

\section{Statistical Analysis}

The study was registered with IBM SPSS 20.0 for Windows. Numerical values were given as mean, minimum and maximum.

\section{Results}

A total of 30 patients, who were admitted to Departments of Pediatrics of Ege University School of Medicine between December 2015 and June 2016, and whose blood cultures had Gram-negative isolation, were included into this study. Fourteen (46.6\%) of these patients were girls while 16 (53.4\%) were boys. Median age was found to be 28 months ( 7 days-16 years). Fifteen (50\%) of the cases were under two years of age (Table I). Fourteen (47\%) of the patients were from the Hematology-Oncology-Bone Marrow Transplantation (BMT) Unit; eight patients (27\%) were from the Department of Pediatric Gastroenterology; three patients (10) were from the ICU; two patients (6\%) were from the Neonatal ICU; and the remaining three (10\%) were from the departments of pediatric pulmonologyallergy and cardiology respectively.

When the patients were evaluated according to their primary diagnoses, the highest group with 16 patients (53.3\%) were immunocompromised patients with hematologic oncology malignancies. According to the 
location of the primary infections, 15 (50\%) patients had catheter-related BSIs (CRBSIs), 12 (40\%) had sepsis and 3 (10\%) had pneumonia (Table I).

Eleven (36.6\%) of the patients were neutropenic. Twenty of the 30 patients $(66.6 \%)$ had central venous catheter and 15 of these patients (75\%) had CRBSIs. The catheters of 7 out of 15 CRBSI patients (46.6\%) were removed due to Gram-negative isolation while the remaining 10 (53.4\%) patients had to continue using the catheter due to various reasons (no other venous access, total parental nutrition, etc.).

Five patients (16.6\%) developed septic shock and 2 $(6.6 \%)$ were lost due to septic shock/ being unresponsive to inotropic agents.

When the distribution of agents in blood cultures identified with conventional methods were evaluated, 12 $(40 \%)$ of them were Klebsiella spp. (11 K. pneumoniae and 1 K. oxytoca); 6 (20\%) were Acinetobacter spp.; 5 (16.6\%) were E.coli; 4 (13.3\%) were Enterobacter cloacae spp.; 1 (3.3\%) was Pantoea agglomerans; 1 (3.3\%) was Citrobacter amalonaticus and 1 (3.3\%) was Pseudomonas aeruginosa (Figure 1).

The identification rates of agents with the microarray method were 100\% (12/12) for Acinetobacter spp., $100 \%$ (5/5) for E. coli, $80 \%$ (4/5) for Enterobacter cloacae spp., 100\% (1/1) for Pseudomonas aeruginosa and 100\% (1/1) for Citrobacter amalonaticus. The identification rate was found to be $100 \%$ except Enterobacter cloacae spp. Since Pantoea agglomerans is a rare agent which is not included in the identification range of the Verigene Gram-negative (BC$\mathrm{GN}$ ) microarray test, we can evaluate positive predictive

Table I. Demographic and clinical characteristics of the patients

\begin{tabular}{|l|l|}
\hline & $\mathbf{n}$ \\
\hline $\begin{array}{l}\text { Gender } \\
\text { Mirl }\end{array}$ & $\mathbf{1 4}(\mathbf{4 6 . 6 \% )}$ \\
\hline $\begin{array}{l}\text { Median age } \\
\text { <2 years }\end{array}$ & $\mathbf{1 6}(\mathbf{5 3 . 4 \% )}$ \\
\hline Primary disease & 28 months (7 days-16 years) \\
Hematological malignancy & $15(50 \%)$ \\
Oncologic malignancy & \\
Bone marrow transplantation & $6(20 \%)$ \\
Other hematologic diseases & $5(16.6 \%)$ \\
Short bowel & $5(16.6 \%)$ \\
Immunodeficiency & $3(10 \%)$ \\
Prematurity & $7(23.3 \%)$ \\
\hline Infection focus & $2(6.6 \%)$ \\
CRBSI & $2(6.6 \%)$ \\
sepsis & \\
pneumonia & $15(50 \%)$ \\
\hline
\end{tabular}

CRBSI: catheter-related bloodstream infections, n: Number values of 29 , but not 30 , samples, and hence, it was possible to correctly identify 28 out of 29 (96.5\%) Gram-negative bacterial agents (Tables II, III, IV, V, VI). The test results were obtained within an average of 114.2 minutes (minimum: 113, maximum: 115, median: 114 minutes) with the microarray method.

With the classic culture method, the positivity of extended-spectrum beta-lactamases (ESBLs) were found to be 7 (58.3\%) in Klebsiella spp. and 2 (40\%) in E. coli while carbapenem resistance was $2(16.6 \%)$ in Klebsiella spp. and 1 (16.6\%) in Acinetobacter spp. As with the microarray method, among ESBL-positive Klebsiella spp., 5 had CTX-M and 1 had OXA; one species with carbapenem-resistance had both OXA and CTX-M; one of ESBL-positive E. coli species had CTX-M; and Acinetobacter species with carbapenemresistance had OXA-resistant genes respectively (Tables II, III, IV, V, VI). As a result, 9 out of a total of 12 (75\%) species, which had ESBL and carbapenem resistances with the classic culture method, were also found to have related resistant genes identified within a relatively short time frame. The presence of CTX-M was defined to be the condition most responsible for resistance.

\section{Discussion}

Nosocomial infections due to Gram-negative bacteria, especially BSIs, have become a globalized problem nowadays. In the United States, BSIs are reported to be one of the most prominent causes of death (7). Due to narrow treatment availability, ESBL-positive Gram-negative bacteria, especially carbapenem-resistant ones, are of great importance for increased morbidity and mortality (8). Hospital infections due especially to carbapenemresistant bacteria have increased greatly around the world in recent years (8). Therefore, it is important to know the frequency of resistant genes in carbapenem-resistant bacteria and multi-drug resistant bacteria. In our country,

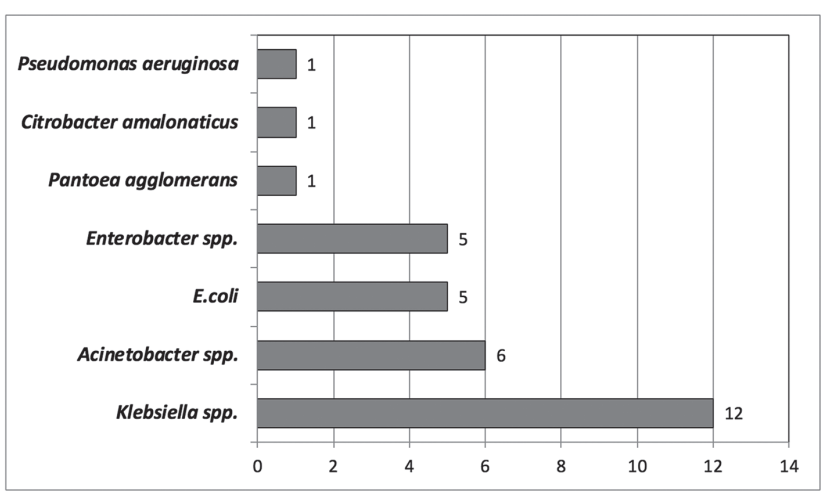

Figure 1. Distribution of Gram-negative bacteria 
Table II. Comparison of the classical culture method and microarray method in terms of detecting Klebsiella spp.

\begin{tabular}{|c|c|c|c|}
\hline no & Culture & Microarray & $\begin{array}{l}\text { Resistance determined } \\
\text { by microarray }\end{array}$ \\
\hline 1. & K. pneumoniae ESBL (+) & K. pneumoniae & CTX-M \\
\hline 2. & K. pneumoniae ESBL (+) & K. pneumoniae & OXA \\
\hline 3. & K. pneumoniae ESBL (+) & K. pneumoniae & CTX-M \\
\hline 4. & K. pneumoniae ESBL (+) & K. pneumoniae & CTX-M \\
\hline 5. & K. pneumoniae ESBL (+) & K. pneumoniae & CTX-M \\
\hline 6. & K. pneumoniae ESBL (+) & K. pneumoniae & CTX-M \\
\hline 7. & K. pneumoniae $\mathrm{ESBL}(+)$ & K. pneumoniae & $\varnothing$ \\
\hline 8. & K. pneumoniae Carbapenem resistant & K. pneumoniae & OXA, CTX-M \\
\hline 9. & K. pneumoniae Carbapenem resistant & K. pneumoniae & $\varnothing$ \\
\hline 10. & K. pneumoniae Amp resistant, Cefuroxime sensitive & K. pneumoniae & $\varnothing$ \\
\hline 11. & K. pneumoniae Amp resistant, Cefuroxime sensitive & K. pneumoniae & $\varnothing$ \\
\hline 12. & K. oxytoca Amp resistant, Cefuroxime sensitive & K. pneumoniae & $\varnothing$ \\
\hline
\end{tabular}

Table III. Comparison of the classical culture method and microarray method in terms of the detection of Acinetobacter spp.

\begin{tabular}{|l|l|l|l|}
\hline no & Culture & Microarray & $\begin{array}{l}\text { Resistance determined } \\
\text { by microarray }\end{array}$ \\
\hline 1. & Acinetobacter spp. Carbapenem resistant & Acinetobacter spp. & OXA \\
\hline 2. & Acinetobacter spp. & Acinetobacter spp. & $\varnothing$ \\
\hline 3. & Acinetobacter spp. & Acinetobacter spp. & $\varnothing$ \\
\hline 4. & Acinetobacter spp. & Acinetobacter spp. & $\varnothing$ \\
\hline 5. & Acinetobacter spp. & Acinetobacter spp. & $\varnothing$ \\
\hline 6. & Acinetobacter spp. & Acinetobacter spp. & $\varnothing$ \\
\hline
\end{tabular}

Table IV. Comparison of the classical culture method and microarray method for the detection of Escherichia coli

\begin{tabular}{|l|l|l|l|}
\hline no & Culture & Microarray & $\begin{array}{l}\text { Resistance determined } \\
\text { by microarray }\end{array}$ \\
\hline 1. & E. coli ESBL (+) & E. coli & CTX-M \\
\hline 2. & E. coli ESBL (+) & E. coli & $\varnothing$ \\
\hline 3. & E. coli & E. coli & $\varnothing$ \\
\hline 4. & E. coli & E. coli & $\varnothing$ \\
\hline 5. & E. coli & E. coli & $\varnothing$ \\
\hline ESBL: Extended spectrum beta-lactamase & & \\
\hline
\end{tabular}

Table V. Comparison of the classical culture method and microarray method for the detection of Enterobacter cloacae spp.

\begin{tabular}{|l|l|l|l|}
\hline no & Culture & Microarray & $\begin{array}{l}\text { Resistance determined } \\
\text { by microarray }\end{array}$ \\
\hline 1. & Enterobacter cloacae spp. & Enterobacter cloacae spp. & $\varnothing$ \\
\hline 2. & Enterobacter cloacae spp. & Enterobacter cloacae spp. & $\varnothing$ \\
\hline 3. & Enterobacter cloacae spp. & Enterobacter cloacae spp. & $\varnothing$ \\
\hline 4. & Enterobacter cloacae spp. & Not detected & $\varnothing$ \\
\hline
\end{tabular}




\begin{tabular}{|l|l|l|l|}
\hline \multicolumn{2}{|l|}{ Table VI. Comparison of the classical culture method and microarray method to detect other rare Gram-negative bacteria } \\
\hline no & Culture & Microarray & $\begin{array}{l}\text { Resistance determined by } \\
\text { microarray }\end{array}$ \\
\hline 1. & Pseudomonas aeruginosa & Pseudomonas aeruginosa & $\varnothing$ \\
\hline 2. & Citrobacter amalonaticus & Citrobacter amalonaticus & $\varnothing$ \\
\hline 3. & Pantoea agglomerans & Not detected & $\varnothing$ \\
\hline
\end{tabular}

there is not enough data on the prevalence of carbapenemresistant infections and resistance levels especially in pediatric patients. In a study carried out in our country, the incidence of bacteremia and fungemia in pediatric patients were evaluated. It was reported that $22.7 \%$ of the cases were due to Gram-negative agents; the most common causative agent was K. pneumoniae; and the most common department with positive blood culture was the pediatric hematology department (9). Similarly, our study found that the most common agent was K. pneumoniae, and the most common patients were immunocompromised ones in the hematology-oncology department.

Serious Gram-negative infections and hence increased mortality and morbidity, alongside late results with classic culture methods, false positive probabilities and false negatives due to insufficient blood samples especially in children and neonates, raise the importance of rapid diagnostic tests nowadays. Many studies in adult patients have been done in this field. There are a few studies in pediatric patients using rapid diagnostic tests such as MALDI-TOF MS, Fluorescence in situ hybridization (FISH) and microarray. Many studies reported the sensitivity and specificity of bacterial identification with the microarray BC-GN method to be $81-100 \%$ or $98-100 \%(10,11,12)$. It was also reported that the identification chance was higher in fresh blood samples than frozen ones.

In a multi-center study, which evaluated identifications of Gram-negative agents with the microarray BC-GN method in BSIs of pediatric patients, sensitivity was found to vary with causative agents as follows: $100 \%$ for Acinetobacter spp., P. aeruginosa and S. Marcescens, 5/6 for Citrobacter spp., 13/14 for Enterobacter spp., 23/24 for E. coli, 2/3 for K. oxytoca, 16/17 for K. pneumoniae and 0/1 for Proteus spp. (13). A study by Dodemont et al. (9) showed a true identification rate of $97.4 \%$ for Gram-negative agents with the microarray method.

In our study, Klebsiella spp., Acinetobacter spp., E. coli, Citrobacter spp. and Pseudomonas aeruginosa were identified $100 \%$ correctly; but only 4 out of 5 isolations of Enterobacter cloacae spp. could be identified. Since Pantoea agglomerans is a non-identifiable agent in the microarray BC-GN method, one sample was removed from the study. Twenty-eight out of the remaining 29 (96.5\%) Gram-negative agents were identified correctly with the microarray method compared to the gold standard of the classic culture method. In our study, Serratia marcescens and Proteus spp., which are normally among the six identifiable Gram-negative agents with microarray, were neither isolated in the classic culture method nor detected in the microarray method.

The biggest superiority of the microarray test over other rapid diagnostic tests such as MALDI-TOF MS is the ability to define six genetic markers concerning with resistance development to multiple beta-lactam antibiotics. It is important to define CTX-M type alongside various carbapenemases. Around the globe, more and more CTX-M rates are being reported among Enterobacteriaceae spp. $(14,15)$. In our study, 9 out of $12(75 \%)$ species with ESBL and carbapenem resistances were found to have resistancerelated genetic predisposition, and CTX-M was found to be the most common among resistant bacteria.

Ease of use with a short training period, little need for experience, simple usage, rapid results ( $<2$ hours), coverage of Gram-negative agents that are the most common agents for nosocomial infections, determination of carbapenemases responsible for resistance together with the bacteria itself within a short time, and hence the ability to start early and effective target-specific antibiotic treatment are the most important advantages of the microarray method $(16,17)$. Early identification of resistant Gram-negative agents especially in immunosuppressive and neutropenic patients, and with early treatment or change in treatment (such as change of antibiotic or central line removal) is life-saving as it can prevent probable septic shock and multiple organ failure. Early agent identification can also make the duration of empiric combination antibiotic therapy shorter, and so long-term unnecessary usage can be avoided. Coverage of Acinetobacter spp., the most common nosocomial infectious agent that can develop multiple drug resistance, is also an important clinical advantage. However, a lack of coverage for agents such as Neisseria meningitidis and 
Haemophilus influenzae, which needs urgent treatment and are diagnosed rapidly with some tests [such as FilmArray blood culture method (BioFire Diagnostic-BCID)], not being in routine use in several hospitals due to high cost, being dependent on positive blood culture and gram staining can be counted as the disadvantages of this method (17).

In conclusion, The Verigene Gram-negative blood culture nucleic acid test (BC-GN; Nanosphere, USA) is a useful assay which accurately and rapidly determines common Gramnegative bacteria and resistance genes from positive blood cultures. The high sensitivity of the test in the pediatric population is demonstrated in this study and we think it has an important role in sepsis management allowing for improved infection control and also early and targeted antibiotic selection. De-escalation can also result in a decrease in the total cost of care for patients with Gramnegative BSIs. Further research and development focusing on the expansion of the panel of the test to allow for routine use and clinical benefit is necessary.

\section{Ethics}

Ethics Committee Approval: With the decision of the Board of Ethics of Ege University, dated 15.04.2015 and numbered 15-3.2 / 7, permission was obtained for this study.

Informed Consent: Informed consent was obtained from the patients included in the study.

Peer-review: Externally peer-reviewed.

\section{Authorship Contributions}

Data Collection or Processing: G.A., M.S., Ş.A., D.Y.K., Z.Ş.B., S.A., B.K., F.Ö., Analysis or Interpretation: M.S., Ş.A., Literature Search: D.Y.K., Z.Ş.B., S.A., B.K., F.Ö., F.V., Writing: G.A.

Conflict of Interest: No conflict of interest was declared by the authors.

Financial Disclosure: This research was supported by Ege University Scientific Research Fund.

\section{References}

1. Al-Hasan MN, Huskins WC, Lahr BD, Eckel-Passow JE, Baddour LM. Epidemiology and outcome of Gram-negative bloodstream infection in children: a population-based study. Epidemiol Infect 2011;139:791-6.

2. Marchaim D, Gottesman T, Schwartz O, et al. National multicenter study of predictors and outcomes of bacteremia upon hospital admission caused by Enterobacteriaceae producing extended-spectrum $\nabla$-lactamases. Antimicrob Agents Chemother 2010;54:5099-104.

3. Schwaber MJ, Carmeli Y. Mortality and delay in effective therapy associated with extended-spectrum beta-lactamase production in Enterobacteriaceae bacteraemia: a systematic review and meta-analysis. I Antimicrob Chemother 2007;60:913-20.

4. Matsuda K, Iwaki KK, Garcia-Gomez I, et al. Bacterial identification by $16 \mathrm{~S}$ rRNA gene PCR-hybridization as a supplement to negative culture results. I Clin Microbiol 2011;49:2031-4.

5. Anthony RM, Brown TJ, French GL. Rapid diagnosis of bacteremia by universal amplification of 235 ribosomal DNA followed by hybridization to an oligonucleotide array. I Clin Microbiol 2000;38:781-8.

6. Wojewoda CM, Sercia L, Navas M, et al. Evaluation of the Verigene Gram-positive blood culture nucleic acid test for rapid detection of bacteria and resistance determinants. I Clin Microbiol 2013;51:2072-6.

7. Wheeler AP, Bernard GR. Treating patients with severe sepsis. N Engl J Med 1999:21;340:207-14.

8. Soysal A. Perikardit ve Endovasküler Enfeksiyonlarda Antibiyotik Kullanımı. 8. Ulusal Çocuk Enfeksiyon Hastalıkları Kongresi Konuşma ve Bildiri Özetleri Kitabı 2013;51-2.

9. Dodémont $M$, De Mendonça $R$, Nonhoff $C$, Roisin $S$, Denis $O$. Performance of the Verigene Gram-negative blood culture assay for rapid detection of bacteria and resistance determinants. I Clin Microbiol 2014;52:3085-7.

10. Hill JT, Tran KD, Barton KL, Labreche MJ, Sharp SE. Evaluation of the nanosphere Verigene BC-GN assay for direct identification of gram-negative bacilli and antibiotic resistance markers from positive blood cultures and potential impact for more-rapid antibiotic interventions. I Clin Microbiol 2014;52:3805-7.

11. Tojo M, Fujita T, Ainoda Y, et al. Evaluation of an automated rapid diagnostic assay for detection of Gram-negative bacteria and their drug-resistance genes in positive blood cultures. PLoS One 2014:4;9:e94064.

12. Sullivan KV, Deburger B, Roundtree SS, Ventrola CA, BleckerShelly DL, Mortensen JE. Pediatric multicenter evaluation of the Verigene gram-negative blood culture test for rapid detection of inpatient bacteremia involving gram-negative organisms, extended-spectrum beta-lactamases, and carbapenemases. I Clin Microbiol 2014;52:2416-21.

13. Carrër A, Nordmann P. CTX-M-15-producing Klebsiella pneumoniae: a change in the epidemiology of ESBL. Pathol Biol (Paris) 2011;59:e133-5.

14. Rodriguez-Villalobos $\mathrm{H}$, Bogaerts $\mathrm{P}$, Berhin $\mathrm{C}$, et al. Trends in production of extended-spectrum beta-lactamases among Enterobacteriaceae of clinical interest: results of a nationwide survey in Belgian hospitals. I Antimicrob Chemother 2011;66:3747.

15. Ledeboer NA, Lopansri BK, Dhiman N, et al. Identification of Gram-Negative Bacteria and Genetic Resistance Determinants from Positive Blood Culture Broths by Use of the Verigene Gram-Negative Blood Culture Multiplex Microarray-Based Molecular Assay. I Clin Microbiol 2015;53:2460-72.

16. Altun $\mathrm{O}, \mathrm{Almuhayawi} M$, Ullberg $\mathrm{M}$, Özenci V. Rapid identification of microorganisms from sterile body fluids by use of FilmArray. I Clin Microbiol 2015;53:710

17. Blaschke AJ, Heyrend C, Byington CL, et al. Rapid identification of pathogens from positive blood cultures by multiplex polymerase chain reaction using the FilmArray system. Diagn Microbiol Infect Dis 2012;74:349-55. 